\section{Symptomscore bei Rhinosinusitis zuverlässig wie CT?}

\section{Bei Erwachsenen mit chronischer Rhinosinusitis decken sich symptomorientierte Skalenwerte und Befunde in der Computertomografie kaum. Bei Kindern stellt sich dieser Zusammenhang jedoch anders dar.}

$\mathrm{D}$ ie chronische Rhinosinusitis zählt zu den häufigsten Erkrankungen im HNO-Bereich. Um die Effektivität von medikamentösen und chirurgischen Behandlungen zu erfassen, sind symptomund lebensqualitätsbezogene Einstufungen der Beschwerden wichtig. Bei Erwachsenen wird in den USA die Belastung der Patienten mit dem Sino-NasalOutcome Test (SNOT-20) erfasst. Studien haben aber gezeigt, dass dieser subjektive Score nicht mit objektiven CTBefunden übereinstimmt.

Bei Kindern mit chronischer Rhinosinusitis findet der SN-5-Test Anwendung. Er umfasst die Bereiche infektiöse Symptome, nasale Obstruktion, allergische Symptome, emotionale Befindlichkeit und Einschränkungen im Alltag.
An einer aktuellen Studie nahmen 32 Kinder im Alter zwischen zwei und zwölf Jahren teil. Sie litten alle an chronischer Rhinosinusitis, die trotz maximaler medikamentöser Therapie weiter bestand. $34 \%$ der jungen Patienten hatten Allergien, 38\% litten an Asthma bronchiale. Kinder mit Immundefizienz, zystischer Fibrose oder Störungen der Ziliarfunktion nahmen nicht an der Untersuchung teil. Bei den Kindern erfolgte eine Einstufung gemäß dem SN-5. Es wurden CT-Bilder der Nasennebenhöhlen angefertigt und die Befunde gemäß der Methode nach Lund-MacKay in einem Score-Wert ausgedrückt.

Zwischen den beiden Scores bestand für alle Kinder eine signifikante positive Korrelation (Koeffizient nach Spearman: $0,68 ; \mathrm{p}<0,001)$. Interessant war, dass ein signifikanter positiver Zusammenhang auch für zahlreiche Untergruppen bestand: Kinder unter sechs Jahren, Kinder ab sechs Jahren, Kinder ohne Allergien, Kinder mit Allergien sowie Kinder ohne Asthma. Lediglich bei der Gruppe der Studienteilnehmer mit Asthma bronchiale ergab sich kein signifikanter $\mathrm{Zu}$ sammenhang.

Fazit: Nach der vorliegenden Studie besteht - im Gegensatz zu Erwachsenen - für Kinder eine signifikante positive Korrelation zwischen dem Rhinosinusitis-Symptomscore SN-5 und dem CTBefund entsprechend dem LundMacKay-Score. Nur für Kinder mit Asthma galt dieser Zusammenhang nicht.

Terrell AM et al. Correlation between $\mathrm{SN}-5$ and computed tomography in children with chronic rhinosinusitis.

Laryngoscope 2009;119:1394-8

\section{Kann man Tinnitus dauerhaft wegtrainieren?}

\author{
In der Behandlung des Tinnitus hat sich die Tinnitus-Retraining-Therapie \\ etabliert, doch wie lange hält ihr therapeutischer Effekt an? Ein \\ italienisches Autorenteam überprüfte die Effizienz der Behandlung \\ bis zu 36 Monate nach Ende der Therapie.
}

$\mathrm{D}$ ie Tinnitus Retraining Therapie (TRT) basiert auf dem neurophysiologischen Modell von Jastreboff, der das subjektive Lautheitsempfinden des Tinnitus als therapeutisch beeinflussbaren negativen Lernprozess verstand. Die Behandlung umfasst medizinische Beratung und Aufklärung (Counseling), psychologische Betreuung und Hörtherapie, bei Bedarf inklusive Tinnitus-Masker.

Wie lange allerdings der therapeutische Effekt anhält, ist nur in wenigen Studien untersucht. Aus diesem Grund führten italienische Forscher eine Studie durch, in der sie den Behandlungserfolg nicht nur zum Ende einer 18-monatigen TRT, sondern auch weitere 18 Monate danach auswerteten. In die Studie waren 45 er- wachsene Patienten aufgenommen worden. Alle hatten mindestens sechs Monate lang an idiopathischem Tinnitus mit oder ohne Hyperakusis - gelitten und waren bislang nicht wegen der Erkrankung behandelt worden.

Während der 18-monatigen Behandlung zeigten sich signifikante Therapieerfolge: Der mittlere Wert des Tinnitus Handycap Inventory (THI) ging um 20 Punkte zurück. Der Therapieeffekt hielt auch in den 18 Monaten nach Ende der Behandlung an. Der Anteil der Patienten, die über eine Abnahme der Beeinträchtigungen in verschiedenen Aktivitätsbereichen (Konzentration, Schlaf, Erholung, Arbeit, soziale Aktivitäten) berichteten, nahm nach Ende der Therapie sogar weiter zu. Von den Patienten bei- spielsweise, die zu Beginn der Behandlung angegeben hatten, sich aufgrund des Tinnitus nicht erholen zu können, gaben $60 \%$ nach 36 Monaten an, keine Probleme mehr zu haben - bei $40 \%$ trat der Effekt erst nach Ende der Therapie ein. Nur ein Patient verschlechterte sich nach Therapieende im Rahmen einer Depression und musste einer psychiatrischen Behandlung zugeführt werden.

Fazit: In dieser Langzeituntersuchung erwies sich die Tinnitus Retraining Therapie als erfolgreich. Die Behandlungserfolge nahmen im zeitlichen Verlauf nach Ende der Behandlung nach 18 Monaten sogar noch weiter zu.

Forti $S$ et al. Are results of tinnitus retraining therapy maintained over time?

Audiol Neurotol 2009; 14: 286-9 